\title{
Melting Behavior of Aliphatic and Aromatic Diamides
}

\section{INTRODUCTION}

Aromatic polyamides form a special class of polymers which have high melting temperatures and yield fibers with very high Youngs moduli. ${ }^{1-3}$ The melting behavior of aliphatic, aliphatic-aromatic, and aromatic polyamides have previously been studied. ${ }^{3-6}$ In general it can be said that the melting temperature $T_{m}$ increases with increasing aromaticity. Black and Preston' ${ }^{3}$ suggest that an important role is played by the ability of a 1,4-substituted benzene unit in the polyamide chain to form a molecular arrangement which allows better intermolecular registration of the amide groups for hydrogen bonding. According to them, one must take into account the possible conjugation of the aromatic group with the amide group which results in the planarity of a larger segment. Aubineau, ${ }^{4}$ studying aliphatic-aromatic copolyamides, observed a considerable rise in $T_{m}$ when phenyl groups were attached to both sides of the amide group. He explained the increase in $T_{m}$ on increasing aromaticity from aliphatic to aliphatic-aromatic by the increase in molecular stiffness. The strong increase in $T_{m}$ on increasing aromaticity from aliphatic-aromatic to aromatic systems is explained by a further increase in molecular stiffness of the polymer chain, the conjugation over the amide bond, and possibly also an increase in hydrogen bonding interaction. Recently Northolt ${ }^{7}$ reported the crystalline structure of poly- $p$-phenyleneterephthalamide. The hydrogen bond distance was found to be 3.04 $\AA$, which is greater than the hydrogen bond distance ${ }^{8}$ in aliphatic polyamides $(2.8 \AA)$. This suggests that poly- $p$-phenyleneterephthalamide has weaker hydrogen bonding than the aliphatic polyamides.

As most of the reported properties of the semicrystalline polyamides depend on the not so well defined crystalline phase, it would be informative to look at simple model compounds. Therefore we studied the effects of substituting a 1,4-tetramethylene group for a 1,4-benzene group in diamides with either butyl or phenyl end groups.

\section{EXPERIMENTAL}

The diamides (Table I) were prepared from purified amines and acid chlorides ${ }^{9}$ and recrystallized from benzyl alcohol. The melting behavior was studied by DSC (Perkin-Elmer DSC-1B). The heating rate was $16^{\circ} \mathrm{C} / \mathrm{min}$. The onset of melting was taken as the melting point $T_{m}$. The heat of melting $\left(\Delta H_{m}\right)$ was obtained by integrating the melting curve with a Kipp integrator connected to the recorder, calibration of the apparatus being run against indium. The entropy of melting $\Delta S_{m}$ was calculated from the enthalpy of melting and the melting temperature:

$$
\Delta S_{m}=\Delta H_{m} / T_{m}
$$

The thermal data obtained are presented in Table I and Figure 1.

\section{RESULTS}

The melting temperatures of the wholly aliphatic (I, II), aliphatic-aromatic-aliphatic (III, IV), and wholly aromatic-diamides (VII, VIII) are independent of the direction of the amide bonds. However, among the aromatic-aliphatic-aromatic diamides (V, VI), dianilinoadipamide (VI) has a higher melting temperature than tetramethylenebisbenzamide (V). The $T_{m}$ of the diamides increased on substituting a 1,4-tetramethylene unit for a 1,4-benzene group. The largest increase in $T_{m}$ is found when the diamide has phenyl end groups, in going from aromatic-aliphatic-aromatic (V, VI) to wholly aromatic compounds (VII, VIII). These results are in agreement with those obtained with aliphatic, aliphatic-aromatic, and wholly aromatic polyamides. ${ }^{4}$

The heats of melting $\left(\Delta H_{m}\right)$ of the wholly aliphatic and aliphatic-aromatic-aliphatic diamides (III, IV) are very similar. A greater difference exists between V and VI and also VII and VIII. Such differences in $\Delta H_{m}$ may be due to a change in molecular interactions, probably as a result of different chain packings. ${ }^{10}$ In going from wholly aliphatic (I, II) to aliphatic-aromatic-aliphatic (III, IV), 
TABLE I

Melting Data for Diamides

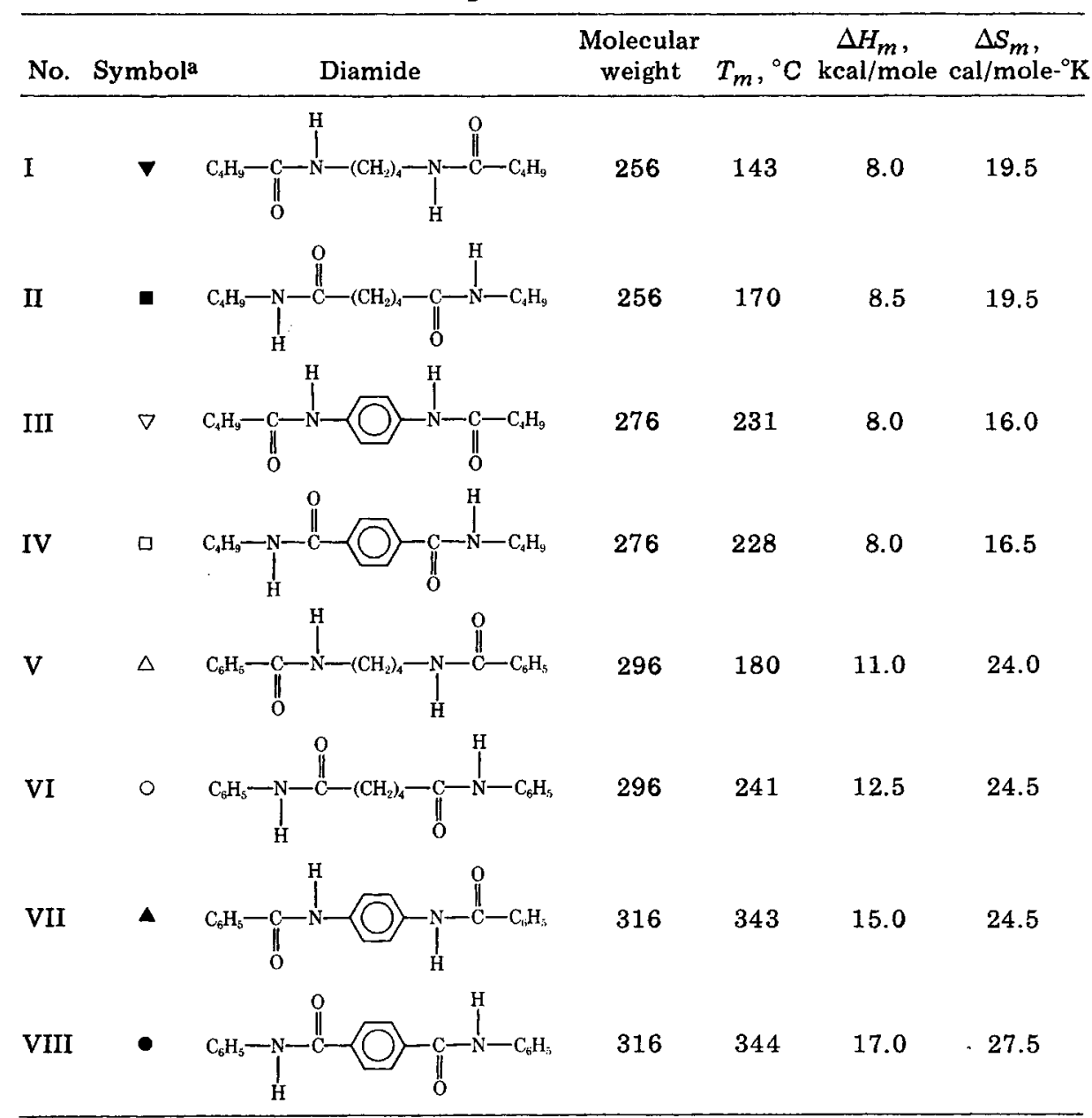

a See Fig. 1.

$\Delta H_{m}$ remains constant. However, $\Delta H_{m}$ increases strongly in going from aromatic-aliphatic-aromatic (V, VI) to wholly aromatic diamides (VII, VIII). This increase is hard to explain, as one would not expect a closer packing in the amide plane. Possibly, this increase in $\Delta H_{m}$ is due to $\pi-\pi$ interactions. The entropy of melting ( $\Delta S_{m}$ ) is very similar for I and II, III and IV, and V and VI. The $\Delta S_{m}$ of VII and VIII show a marked difference which has not been explained yet.

The $\Delta S_{m}$ decreased in going from wholly aliphatic to aliphatic-aromatic-aliphatic. This must be due to the chain-stiffening effect of incorporating a benzene unit in the diamides. In going from aromatic-aliphatic-aromatic to wholly aromatic, the $\Delta S_{m}$ increases slightly. It had been expected that $\Delta S_{m}$ would decrease due to of a further increase in molecular stiffness as a result of a increase in aromaticity and possible conjugation.

\section{CONCLUSION}

The melting temperatures of the diamides increase with increasing aromaticity, as in the aliphatic, aliphatic-aromatic, and aromatic polyamides. 4 This increase is strongest when the 1,4-tetramethylene is substituted for a 1,4-benzene unit in diamide with phenyl end groups.

The increase in $T_{m}$ from wholly aliphatic (I, II) to aliphatic-aromatic-aliphatic (III, IV) is due 
${ }_{\mathrm{m}}$

$\left({ }^{\circ} \mathrm{C}\right)$

\section{$\Delta \mathrm{H}_{\mathrm{m}}$}

$\mathrm{kcal} / \mathrm{mol}$

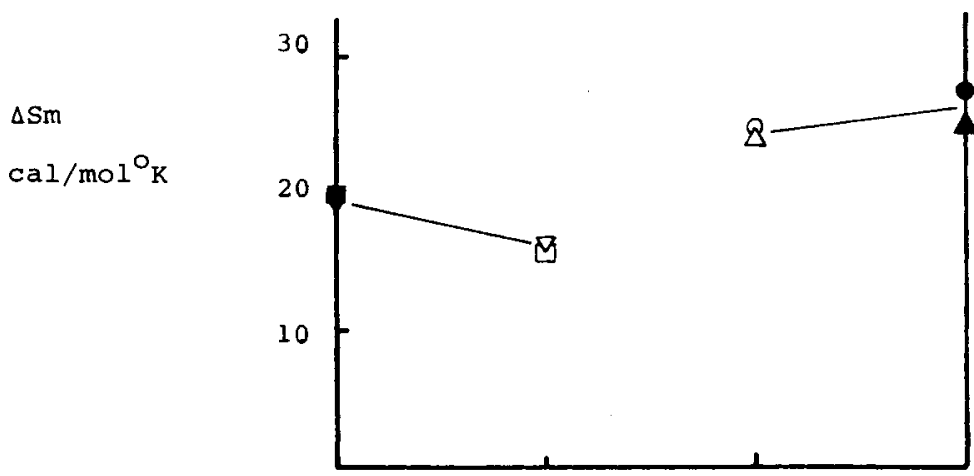

Fig. 1. Melting behavior of diamides: ( $\nabla)$ I and $(\square)$ II, wholly aliphatic; $(\nabla)$ III and ( $\square$ ) IV, ali-

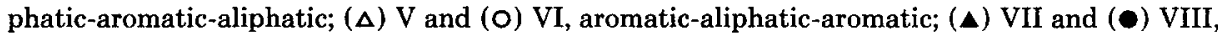
wholly aromatic diamides. 
solely to a decrease in $\Delta S_{m}$, which results from the chain stiffening due to incorporation of a benzene unit in the diamide. The strong increase in $T_{m}$ from aromatic-aliphatic-aromatic (V, VI) to wholly aromatic compounds (VII, VIII) seems to be due to the high heats of fusion of the wholly aromatic compounds.

The conjugation which is expected to take place in the aromatic polyamides ${ }^{3,4}$ does not seem to take place in these aromatic diamides. Conjugation reduces the intermolecular interaction and thus both $\Delta H_{m}$ and $\Delta S_{m}$. Neither effect seems to take place between the aromatic-aliphatic-aromatic (V, VI) and the wholly aromatic diamides (VII, VIII).

\section{References}

1. J. H. Rothuizen, Text. Inst. Ind., 11, 142 (1973).

2. R. E. Wilfong and J. Zimmerman, J. Appl. Polym. Sci., 17, 2039 (1973).

3. J. Preston and W. B. Black, in Man-Made Fibers, H. F. Mark, S. M. Atlas, and E. Cernia, Eds., Vol. 2, Interscience, New York, 1968, p. 365.

4. C. Aubineau, R. Audebert, and G. Champetier, Bull. Soc. Chim. France, 1970, 1404.

5. P. W. Morgan and S. L. Kwolek, Macromolecules, 8, 104 (1975).

6. T. C. Tranter, J. Polym. Sci. A, 2, 4289 (1964).

7. M. G. Northolt, Eur. Polym. J., 10, 799 (1974).

8. C. W. Bunn and E. V. Garner, Proc. Roy. Soc. (London), A189, 39 (1948).

9. A. I. Vogel, Practical Organic Chemistry, 32rd ed., Longmans, London, 1956, p. 361.

10. A. Bondi, Physical Properties of Molecular Crystals, Liquids and Glasses, Wiley, New York-London, 1968, p. 139.

R. J. GaYMANS

S. HARKEMA

Department of Chemical Technology

Twente University of Technology

P.O. Box 217

Enschede, The Netherlands

Received August 24, 1976 